\title{
Reparo artroscópico da lesão de manguito rotador: Uma análise da função, força muscular e dor entre técnicas de fileira simples e fileira dupla
}

\section{Arthroscopic Repair of Rotator Cuff Injury: An Analysis of Function, Muscular Strength and Pain Between Single Row and Double Row Techniques}

\author{
Thiago Medeiros Storti ${ }^{1,2(0)}$ Thiago da Silva Ribeiro $^{3(0)}$ Rafael Salomon Silva Faria1(1) \\ João Eduardo Simionatto ${ }^{1 \odot}$ Carolina Simionatto ${ }^{1 \odot}$ Alexandre Firmino Paniago ${ }^{1,2}{ }^{1}$ \\ ${ }^{1}$ Instituto do Ombro de Brasília, Brasília, DF, Brasil \\ ${ }^{2}$ Hospital Ortopédico e Medicina Especializada, Instituto de Pesquisa \\ e Ensino, Brasília, DF, Brasil \\ 3 Instituto do Ombro de Brasília, Brasília, DF, Brasil \\ Endereço para correspondência Dr. Thiago Medeiros Storti, Hospital \\ Ortopédico e Medicina Especializada (HOME), SGAS Quadra 613 - \\ Conjunto C - Asa Sul Brasília 70200-730, DF, Brasil \\ (e-mail: thiago_storti@hotmail.com).
}

Rev Bras Ortop 2022;57(3):472-479.

\begin{abstract}
Resumo
Objetivo Avaliar os pacientes submetidos ao reparo artroscópico do manguito rotador (MR), comparando-se os resultados funcionais, força muscular e dor obtidos após as técnicas de fileira simples (FS) e de fileira dupla (FD).

Métodos Foram coletados os dados do seguimento pós-operatório (mínimo de 12 meses) de 128 pacientes submetidos ao reparo artroscópico do MR durante o período de 2011 a 2018. As variáveis clínico-funcionais foram coletadas por meio do exame clínico, e as variáveis demográficas, cirúrgicas e das lesões do MR a partir dos prontuários eletrônicos. Os resultados foram comparados entre os grupos FS e FD.

Resultados O grupo FD demonstrou força de elevação anterior maior quando comparado ao grupo FS (FS: 4,72 $\pm 2,73 \mathrm{~kg}$ versus $\mathrm{FD}: 5,90 \pm 2,73 \mathrm{~kg} ; p=0,017$ ). As demais variáveis de

\section{Palavras-chave}

- manguito rotador

- artroscopia

- força muscular

- avaliação funcional

- ombro força muscular, Constant-Murley Score, University of California at Los Angeles Shoulder Rating Scale (UCLA, na sigla em inglês) e dor foram similares. Realizando-se a estratificação por tamanho, na análise das lesões pequenas e médias, não foram encontradas diferenças entre os grupos. Porém, na análise das lesões grandes e extensas, os pacientes submetidos à FD apresentaram superioridade tanto na força muscular de elevação (FS: $3,98 \pm 2,24 \mathrm{~kg}$ versus FD: $6,39 \pm 2,73 \mathrm{~kg}$ ) quanto no escore Constant (FS: $81 \pm 10$ versus FD: $88 \pm 7$ ).
\end{abstract}

\footnotetext{
${ }^{*}$ Trabalho desenvolvido no Hospital Ortopédico e Medicina Especializada, Instituto de Pesquisa e Ensino, Brasília, DF, Brasil.
}

recebido

29 de Julho de 2020

aceito

23 de Abril de 2021

Publicado on-line

de Janeiro 21, 2022
DOI https://doi.org/ 10.1055/s-0041-1735943. ISSN 0102-3616.
(C) 2022. Sociedade Brasileira de Ortopedia e Traumatologia. All rights reserved.

This is an open access article published by Thieme under the terms of the Creative Commons Attribution-NonDerivative-NonCommercial-License, permitting copying and reproduction so long as the original work is given appropriate credit. Contents may not be used for commercial purposes, or adapted, remixed, transformed or built upon. (https://creativecommons.org/ licenses/by-nc-nd/4.0/)

Thieme Revinter Publicações Ltda., Rua do Matoso 170, Rio de Janeiro, RJ, CEP 20270-135, Brazil 


\begin{abstract}

\section{Keywords}

- rotator cuff

- arthroscopy

- muscle strength

- functional evaluation

- shoulder

Objective To evaluate the patients submitted to arthroscopic repair of the rotator cuff (RC), comparing the functional results, muscle strength, and pain obtained after single row (SR) and double row (DR) techniques.

Methods Data were collected at the postoperative follow-up (minimum of 12 months) of 128 patients submitted to arthroscopic RC repair from 2011 to 2018. The clinical-functional variables were collected through the clinical examination, and the demographic, surgical and injury variables of the RC were collected from the electronic medical records. The results were compared between the SR and DR groups. Results The DR group showed higher anterior elevation strength when compared with the SR group (SF: $4.72 \pm 2.73 \mathrm{~kg}$ versus DR: $5.90 \pm 2.73 \mathrm{~kg} ; p=0.017$ ). The other variables of muscle strength, Constant-Murley Score, University of California at Los Angeles Shoulder Rating Scale (UCLA), and pain, were similar. Performing the stratification by size, in the analysis of small and medium injuries, no differences were found between the groups. However, in the analysis of large and extensive injuries, patients submitted to DR presented superiority of both muscle lifting strength (SF: $3.98 \pm 2.24 \mathrm{~kg}$ versus DR: $6.39 \pm 2.73 \mathrm{~kg}$ ) and Constant score (SF: $81 \pm 10$ versus DR: $88 \pm 7)$.

Conclusion The use of the DR technique in arthroscopic RC repair allowed higher levels of muscle strength for anterior shoulder elevation when compared with the SF technique. Data stratification in large and extensive injuries showed superiority of anterior shoulder elevation muscle strength and of the Constant score in patients submitted to DR.
\end{abstract}

Conclusão A utilização da técnica de FD no reparo artroscópico do MR possibilitou maiores níveis de força muscular para elevação anterior do ombro quando comparada à técnica de FS. A estratificação dos dados em lesões grandes e extensas evidenciou superioridade da força muscular de elevação anterior do ombro e do escore Constant nos pacientes submetidos à FD.

\section{Introdução}

As lesões do manguito rotador (MR) são comuns na prática ortopédica, correspondendo a entre 30 e $70 \%$ dos quadros de dor no ombro. ${ }^{1,2} \mathrm{O}$ arsenal terapêutico é amplo, englobando tratamentos conservadores e cirúrgicos. ${ }^{3} 0$ método cirúrgico pode ser realizado por meio da técnica aberta com mínima incisão ("mini open") ou da técnica artroscópica, e, em alguns casos de lesões irreparáveis associadas a artropatia do MR, existe a possibilidade de artroplastia reversa do ombro. ${ }^{3,4}$ No Brasil, segundo dados do Departamento de Informática do Sistema Único de Saúde SUS (DataSUS) e considerando-se tanto o reparo aberto quanto o artroscópico, foram realizadas 50.207 cirurgias de reparo do MR no período de 2003 a $2015 . .^{5}$ No ano de 2015, a taxa de reparos do MR foi de 2,81 procedimentos para cada 100.000 habitantes. ${ }^{5}$ Os dados citados, entretanto, englobam apenas os procedimentos realizados pelo SUS, o que leva a inferir que os números referentes ao reparo do MR em todo o Brasil são ainda maiores, uma vez que os dados referentes à saúde suplementar não são publicamente agregados e divulgados.

Uma vez estabelecido o tratamento por via artroscópica, outro ponto a ser decidido é quanto à técnica de sutura do tendão no úmero, a qual pode ser realizada em fileira simples (FS), fileira dupla (FD), ou equivalente transóssea. No Brasil, a técnica mais difundida é a de FS, utilizada por 50,4\% dos ortopedistas, enquanto $26,1 \%$ utilizam a configuração em FD. ${ }^{6}$ Ao comparar as duas técnicas, a literatura apresenta divergências e os estudos não são conclusivos. ${ }^{7-10}$ DeHaann et al. $^{7}$ e Chen et al. ${ }^{8}$ não evidenciaram diferenças funcionais entre as técnicas. Ying et al. ${ }^{9}$ demonstraram melhor força muscular no grupo submetido à FD. Sobhy et al. ${ }^{10}$ determinaram que, a curto e médio prazo, o grupo da FD apresentou escore UCLA significativamente melhor e que, a longo prazo, há uma correlação direta entre a integridade do MR e os resultados funcionais, com superioridade da FD. No cenário nacional, o trabalho publicado por Senna et al. ${ }^{11}$, não evidenciou diferença funcional estatisticamente significativa entre os dois métodos.

Diante de tais evidências, observa-se que as técnicas de fixação em FS e FD no reparo artroscópico do MR ainda apresentam resultados controversos, principalmente no Brasil, posto que há apenas um estudo nacional sobre o tópico. ${ }^{11}$ Tendo em vista que a utilização de mais âncoras torna o procedimento mais oneroso, ${ }^{12}$ faz-se necessário demonstrar se há real benefício clínico ao lançar mão dessa técnica. Para isso, deve-se ampliar a base de estudos para comprovar a superioridade de uma das técnicas ou a equivalência de ambas.

Desta forma, o objetivo do presente estudo foi avaliar os pacientes submetidos ao reparo artroscópico do MR, 
comparando-se os resultados de força muscular, funcionais e dor obtidos após as técnicas de FS e de FD. Diante do exposto e baseado na lógica que levou ao desenvolvimento da técnica de FD (maior área de inserção do MR - footprint -, gerando menor chance de rerruptura), ${ }^{10,13}$ hipotetizamos que os pacientes submetidos à técnica de FD apresentarão superioridade nos desfechos analisados.

\section{Metodologia}

\section{Design do Estudo e Participantes}

Trata-se de um estudo de coorte retrospectiva. Durante o período de 2011 a 2018, 465 pacientes foram submetidos ao reparo artroscópico do MR. Foram incluídos pacientes $>18$ anos submetidos ao reparo artroscópico do MR e com seguimento pós-operatório mínimo de 12 meses. Foram excluídos aqueles que, durante o ato cirúrgico, foram submetidos a outros procedimentos, como reparo do lábio glenoidal, acromioplastia, tenotomia e tenodese do bíceps, assim como aqueles que se recusaram a participar da pesquisa e cujo contato não foi possível, o que caracterizou perda de seguimento. Após a aplicação dos critérios (inclusão e exclusão), 128 pacientes aceitaram participar da pesquisa e comparecer ao hospital para serem reavaliados, totalizando 135 ombros.

\section{Desfechos de Análise}

Considerou-se como desfecho primário os escores funcionais pós-operatórios, UCLA e Constant, e como desfechos secundários as variáveis de força, amplitude de movimento (ADM) e dor pós-operatórios.

\section{Métodos e Instrumentos}

A coleta de dados durante o seguimento pós-operatório foi realizada em duas etapas: primeira - consulta aos prontuários para coleta de características demográficas, cirúrgicas e das lesões do MR; e segunda - avaliação clínica com coleta de variáveis funcionais, força muscular e dor.

Os dados demográficos e de caracterização da lesão de MR foram obtidos por meio de análise dos prontuários eletrônicos. As variáveis coletadas foram: idade, gênero, dominância e lateralidade, tempo de seguimento, tabagismo, diabetes mellitus, tipo de ruptura (total ou parcial), classificação da lesão (pequena, média, grande ou extensas para as lesões completas, e bursal, articular ou intratendinosa para as lesões parciais), mecanismo da lesão (traumático ou degenerativo), tendões abordados, tipo de fixação (FS ou FD) e número de âncoras utilizadas no tratamento cirúrgico. As lesões completas foram classificadas, conforme a medida de seu maior diâmetro, em pequenas $(<1 \mathrm{~cm})$, médias (de $1 \mathrm{a}$ $3 \mathrm{~cm}$ ), grandes (de 3 a $5 \mathrm{~cm}$ ) e extensas $(\geq 5 \mathrm{~cm}) \cdot{ }^{14,15}$ As lesões extensas, no entanto, podem ser definidas tanto pelo critério mencionado, $\geq 5 \mathrm{~cm}$, quanto pela ruptura completa de $\geq 2$ tendões. ${ }^{15} \mathrm{O}$ tamanho e a classificação das lesões foram obtidos a partir das ressonâncias magnéticas (RMs) pré-operatórias e/ou descrições cirúrgicas. Quando houve divergência entre o laudo da RM e a descrição cirúrgica, considerou-se a descrição intraoperatória.
O segundo momento de avaliação foi realizado presencialmente, quando foram aferidas a força $(\mathrm{kg})$ e a amplitude $\left({ }^{\circ}\right)$ dos movimentos de elevação, rotação lateral e rotação medial do ombro. A força foi aferida por dinamômetro digital e mensurada em $\mathrm{Kg}$, enquanto a amplitude foi aferida por goniometria e mensurada em graus. Além disso, durante esta avaliação, foram aplicados os escores de capacidade funcional reportada University of California at Los Angeles Shoulder Rating Scale (UCLA, na sigla em inglês) ${ }^{16}$ e Constant-Murley Score (Constant), ${ }^{17}$ juntamente à coleta dos dados referentes à dor, utilizando-se a escala visual-analógica. ${ }^{18}$ As escalas mencionadas acima (UCLA e Constant) foram traduzidas para o português e adaptadas culturalmente para a população brasileira $^{19,20}$ e são frequentemente utilizadas para avaliação da função de membros superiores em pacientes com lesões de MR. ${ }^{10,11,21-23}$

Após a tabulação dos dados, os indivíduos foram divididos em dois grupos, com base na técnica de fixação cirúrgica utilizada: FS e FD.

\section{Procedimento Cirúrgico}

As cirurgias foram realizadas por cirurgiões especialistas e com ampla experiência no tema. A fixação em $\mathrm{FS}$ foi realizada através da técnica na qual o tendão é atado, por meio de pontos simples, às âncoras dispostas em uma única fileira. ${ }^{24}$ A técnica de fixação em FD realizada foi a descrita por Lo et al. ${ }^{13}$ e consiste em uma fileira medial de âncoras atadas com pontos em "U" e outra fileira lateral de âncoras atadas com pontos simples. Os procedimentos foram realizados na posição de "cadeira de praia".

SabeSabe-se que, em nosso meio, o custo do material cirúrgico é um fator limitante. A intenção do cirurgião ao solicitar o procedimento ao convênio era sempre realizar a fixação em FD; porém, nem sempre a quantidade de âncoras autorizada era o suficiente para tal. Quando a fixação em FD não era possível, optou-se pela técnica de fileira simples. Portanto, uma vez que todas as lesões selecionadas eram passíveis de tratamento por FD, o fator que direcionou para uma técnica ou outra foi a disponibilidade de materiais.

Todos os pacientes com lesões parciais foram submetidos ao tratamento conservador por, no mínimo, 3 meses. Quando não havia boa resposta a esta terapêutica, indicava-se o tratamento cirúrgico.

Aqueles que apresentaram critérios de irreparabilidade foram submetidos a outras técnicas operatórias que não a artroscópica e, portanto, não foram incluídos no presente estudo.

\section{Reabilitação Pós-operatória}

A reabilitação pós-operatória foi realizada através de manutenção em tipoia tipo Velpeau simples por 6 semanas, sendo estimulada a movimentação ativa do cotovelo, do punho e da mão desde o pós-operatório imediato. Após a 6 $6^{\text {a }}$ semana, foi iniciado o ganho do movimento ativo. Por fim, após a $12^{\mathrm{a}}$ semana, foi iniciado o fortalecimento muscular. 


\section{Aprovações Éticas}

Todos os pacientes assinaram o Termo de Consentimento Livre Esclarecido. $O$ presente estudo foi submetido à avaliação e à aprovação do Comitê de Ética e Pesquisa com Seres Humanos, com número do parecer 2.444.726, CAAE: 80401317.3.0000.0023.

\section{Estatística}

Procedeu-se à análise descritiva, expressa pelas medidas de tendência central e de dispersão adequadas para os dados numéricos e pela frequência e percentual para os dados categóricos. Para as variáveis numéricas, foi realizado inicialmente o teste de normalidade de Shapiro-Wilk. Para as variáveis que não apresentaram distribuição normal, a análise estatística foi realizada por meio do teste não paramétrico de Mann-Whitney, e para aquelas que apresentaram distribuição normal, utilizou-se o teste t de Student. Para as variáveis categóricas, utilizou-se os testes de qui-quadrado ou exato de Fisher, a depender do número de categorias da variável aleatória.

Posteriormente, a amostra foi estratificada nos seguintes subgrupos: 1-lesões pequenas e médias; e 2-lesões grandes e extensas. A partir disso, foram realizadas novas comparações dos resultados funcionais e de força muscular das técnicas de FS e FD dentro destas estratificações.

Também foi realizada análise de regressão linear múltipla para avaliar se as características dos pacientes ou das lesões apresentaram influência nos resultados clínico-funcionais. As variáveis correlacionadas foram: tempo de seguimento, idade, dominância, tamanho da lesão, etiologia da lesão, diabetes e tabagismo.

O critério de determinação de significância adotado foi ao nível de 5\%. A análise estatística foi processada pelo software IBM SPSS Statistics for Windows, versão 20.0 (IBM Corp., Armonk, NY EUA).

\section{Resultados}

\section{Características Demográficas e Cirúrgicas Entre os Grupos}

No período de 2011 a 2018, 465 pacientes foram submetidos ao reparo artroscópico do MR. Destes, 147 foram excluídos por terem sido submetidos a outros procedimentos durante a cirurgia, 8 foram a óbito e 182 se recusaram a participar ou o contato não foi possível.

A amostra foi composta por 135 ombros (128 pacientes). Destes, 94 (69,6\%) correspondem aos casos operados pela técnica de FS e $41(30,4 \%)$ à de FD. 0 tempo médio de seguimento foi de 46,5 meses, sendo que não houve diferença estatisticamente significativa entre os grupos FS e FD. Quando comparadas as características demográficas e o perfil cirúrgico entre os grupos, observou-se diferença estatisticamente significativa para a idade (FS: $59 \pm 9$ versus FD: $55 \pm 8)$, superior no grupo FS $(p=0,010)$ e para o número de âncoras utilizadas (FS: $3 \pm 1$ versus $\mathrm{FD}: 4 \pm 1$ ), superior no grupo $\mathrm{FD}(p=0,012)$. As demais variáveis foram estatisticamente similares e estão expostas na $\boldsymbol{- T a b e l a} \mathbf{1}$.

\section{Características das Lesões de Manguito Rotador Entre os Grupos}

Quanto ao tipo de lesão, ambos os grupos apresentaram predominância dos casos de rupturas totais (FS: $83 \%$ versus FD: $82,9 \%$ ). Dentre as rupturas parciais, as bursais foram maioria e não houve nenhum caso intrassubstancial. Em relação ao tamanho das lesões totais, o grupo FS apresentou maior percentual das lesões extensas (34,6\%), enquanto o grupo FD, das lesões médias (32,4\%). No entanto, estas diferenças entre os grupos quanto ao percentual de cada lesão não apresentaram significância estatística $(p=0,136)$. A descrição completa dos dados está exposta na $\mathbf{- T a b e l a} \mathbf{2}$.

Tabela 1 Caracterização da amostra

\begin{tabular}{|l|l|l|l|l|}
\hline \multirow{2}{*}{ Variável } & Total & Fileira simples & Fileira dupla \\
\cline { 2 - 4 } & $135(100 \%)$ & $94(69,6 \%)$ & $41(30,4 \%)$ \\
\hline Idade (anos) & $58 \pm 9$ & $59 \pm 9$ & $55 \pm 8$ & $24(58,5 \%)$ \\
\hline Gênero Feminino & $77(57 \%)$ & $53(56,4 \%)$ & $0,010^{*}$ \\
\hline Gênero Masculino & $58(43 \%)$ & $41(43,6 \%)$ & $17(41,5 \%)$ \\
\hline Tempo de seguimento (meses) & $46,5 \pm 23,55$ & $44,19 \pm 23,95$ & $50,32 \pm 22,29$ \\
\hline Membro Dominante & $91(67,4 \%)$ & $62(66 \%)$ & $29(70,7 \%)$ & 0,111 \\
\hline Diabéticos & $24(17,8 \%)$ & $20(21,3 \%)$ & $4(9,8 \%)$ & 0,586 \\
\hline Tabagistas & $9(6,7 \%)$ & $7(7,4 \%)$ & $2(4,9 \%)$ & 0,107 \\
\hline Lesão degenerativa & $89(65,9 \%$ & $59(67,8 \%)$ & $30(73,2 \%)$ \\
\hline Lesão traumática & $46(34,1 \%)$ & $35(37,2 \%)$ & $11(26,8 \%)$ \\
\hline Número de tendões abordados:1 & $46(34,1 \%)$ & $32(34 \%)$ & $14(34,1 \%)$ \\
\hline Número de tendões abordados: 2 & $71(52,1 \%)$ & $46(48,9 \%)$ & $25(61 \%)$ \\
\hline Número de tendões abordados: 3 & $18(13,3 \%)$ & $16(17 \%)$ & $2(4,9 \%)$ \\
\hline Número de âncoras & $3,56 \pm 1,17$ & $3 \pm 1$ & $4 \pm 1$ & 0,241 \\
\hline
\end{tabular}


Tabela 2 Caracterização e comparação das lesões de Manguito Rotador (parciais e totais) entre os grupos Fileira Simples (FS) e Fileira Dupla (FD)

\begin{tabular}{|c|c|c|c|c|c|c|}
\hline \multicolumn{3}{|c|}{ Fileira simples } & \multicolumn{3}{|c|}{ Fileira dupla } & \multirow{3}{*}{$\frac{\text { valor-p }}{0,136}$} \\
\hline \multirow{2}{*}{$\begin{array}{l}\text { Parcial } \\
16(17 \%)\end{array}$} & Bursal & $13(81,2 \%)$ & \multirow{2}{*}{$\begin{array}{l}\text { Parcial } \\
7(17,1 \%)\end{array}$} & Bursal & 7 (100\%) & \\
\hline & Articular & $3(18,8 \%)$ & & Articular & 0 & \\
\hline \multirow{4}{*}{$\begin{array}{l}\text { Total } \\
78 \text { (83\%) }\end{array}$} & Pequena & $13(16,7 \%)$ & \multirow{4}{*}{$\begin{array}{l}\text { Total } \\
34(82,9 \%)\end{array}$} & Pequena & $9(26,5 \%)$ & \\
\hline & Média & $22(28,2 \%)$ & & Média & $11(32,4 \%)$ & \\
\hline & Grande & $16(20,5 \%)$ & & Grande & $10(29,4 \%)$ & \\
\hline & Maciça & $27(34,6 \%)$ & & Maciça & $4(11,8 \%)$ & \\
\hline
\end{tabular}

Tabela 3 Comparação das variáveis funcionais, força muscular e dor entre os grupos Fileira Simples (FS) e Fileira Dupla (FD)

\begin{tabular}{|c|c|c|c|c|}
\hline Variável & & Fileira simples & Fileira dupla & valor-p \\
\hline \multirow[t]{2}{*}{ Elevação } & $\operatorname{ADM}\left({ }^{\circ}\right)$ & $157 \pm 29$ & $161 \pm 27$ & 0,302 \\
\hline & Força $(\mathrm{kg})$ & $4,72 \pm 2,73$ & $5,90 \pm 2,73$ & $0,017^{*}$ \\
\hline \multirow[t]{2}{*}{ Rotação Lateral } & $\operatorname{ADM}\left({ }^{\circ}\right)$ & $58 \pm 22$ & $60 \pm 23$ & 0,595 \\
\hline & Força(kg) & $5 \pm 2,66$ & $5,05 \pm 2,09$ & 0,535 \\
\hline \multirow[t]{2}{*}{ Rotação Medial } & $\operatorname{ADM}\left({ }^{\circ}\right)$ & $60 \pm 23$ & $64 \pm 18$ & 0,506 \\
\hline & Força $(\mathrm{kg})$ & $6,28 \pm 3,12$ & $6,97 \pm 2,96$ & 0,165 \\
\hline UCLA & & $33 \pm 3$ & $33 \pm 3$ & 0,365 \\
\hline Constant & & $82 \pm 11$ & $86 \pm 9$ & 0,084 \\
\hline \multirow[t]{4}{*}{ Dor ao seguimento } & Sem dor & $48(51,1 \%)$ & $23(56,1 \%)$ & \multirow[t]{4}{*}{0,759} \\
\hline & Leve (1-3) & $27(28,7 \%)$ & $9(22 \%)$ & \\
\hline & Moderada (4-6) & $15(16 \%)$ & $6(14,6 \%)$ & \\
\hline & Intensa (7-10) & $4(4,3 \%)$ & $3(7,3 \%)$ & \\
\hline
\end{tabular}

Abreviações: ADM, amplitude de movimento; CONSTANT, Constant-Murley Score; UCLA, University of California at Los Angeles Shoulder Rating Scale.

\section{Comparação de Variáveis de Força Muscular, Funcionais e Dor entre os Grupos}

Foi observada uma diferença estatisticamente significativa na força de elevação anterior do ombro entre os grupos $(p=0,017)$. O grupo FD demonstrou maiores níveis de força quando comparado com o grupo FS na elevação anterior (FS: $4,72 \pm 2,73 \mathrm{~kg}$ versus FD: 5,90 $\pm 2,73 \mathrm{~kg}$ ). As demais variáveis de força muscular e função (ADM e questionários de capacidade funcional reportada) foram similares $(p>0,05)$ (-Tabela 3 ).

Quanto à dor no período de seguimento, não houve diferença estatisticamente significativa entre os grupos (-Tabela 3). Porém, foi possível observar que, em ambos os grupos, $>50 \%$ dos pacientes não apresentaram quadro álgico durante a avaliação clínica no seguimento pósoperatório.

A análise de regressão linear múltipla não mostrou relação entre os resultados clínico-funcionais e as variáveis de tempo de seguimento, dominância, tamanho da lesão, etiologia da lesão, diabetes e tabagismo. No entanto, a idade dos pacientes correlacionou-se aos resultados de força de elevação, rotação lateral e rotação medial.

\section{Comparação de Variáveis Funcionais e de Força Muscular Estratificadas Pelo Tamanho das Lesões Totais}

Realizando-se a análise de forma estratificada, comparou-se os resultados funcionais e de força muscular entre os grupos (FS e FD), estratificados em lesões pequenas/médias e lesões grandes/extensas.

$\mathrm{Na}$ análise por lesões pequenas e médias, não foram encontradas diferenças entre os grupos ( - Tabela 4). Porém, na análise das lesões grandes e extensas, os pacientes submetidos à FD apresentaram superioridade tanto na força muscular de elevação (FS: $3,98 \pm 2,24 \mathrm{~kg}$ versus $\mathrm{FD}$ : $6,39 \pm 2,73 \mathrm{~kg}$ ) quanto no escore Constant (FS: $81 \pm 10$ versus FD: $88 \pm 7$ ) (-Tabela 5).

\section{Discussão}

Analisando-se as características da amostra, a única variável que apresentou diferença estatisticamente significativa entre os grupos foi a idade, a qual era maior no grupo de FS. Em ambos, o valor médio foi compatível com a 
Tabela 4 Comparação das variáveis funcionais e de força muscular entre os grupos Fileira Simples (FS) e Fileira Dupla (FD), estratificados nas lesões pequenas e médias do manguito rotador

\begin{tabular}{|l|l|l|l|l|}
\hline \multicolumn{4}{|l|}{ Lesões pequenas e médias do manguito rotador } \\
\hline Variável & & Fileira simples & Fileira dupla & valor-p \\
\hline \multirow{2}{*}{ Elevação } & $\operatorname{ADM~}\left(^{\circ}\right)$ & $160 \pm 28$ & $159 \pm 27$ & 0,993 \\
\cline { 2 - 5 } & Força $(\mathrm{kg})$ & $5,41 \pm 2,89$ & $5,80 \pm 2,94$ & 0,642 \\
\hline \multirow{2}{*}{ Rotação Lateral } & $\operatorname{ADM~}\left(^{\circ}\right)$ & $58 \pm 20$ & $57 \pm 23$ & 0,833 \\
\cline { 2 - 5 } & Força $(\mathrm{kg})$ & $5,39 \pm 2,83$ & $4,95 \pm 2,42$ & 0,772 \\
\hline \multirow{2}{*}{ Rotação Medial } & $\operatorname{ADM~}\left(^{\circ}\right)$ & $62 \pm 21$ & $64 \pm 20$ & 0,712 \\
\cline { 2 - 5 } & Força $(\mathrm{kg})$ & $6,70 \pm 3,06$ & $7,45 \pm 3,19$ & 0,393 \\
\hline UCLA & & $34 \pm 2$ & $33 \pm 3$ & 0,899 \\
\hline Constant & & $86 \pm 8$ & $86 \pm 11$ & 0,927 \\
\hline
\end{tabular}

Abreviações: ADM, amplitude de movimento; CONSTANT, Constant-Murley Score; UCLA, University of California at Los Angeles Shoulder Rating Scale.

Tabela 5 Comparação das variáveis funcionais e de força muscular entre os grupos Fileira Simples (FS) e Fileira Dupla (FD), estratificados nas lesões grandes e extensas do manguito rotador

\begin{tabular}{|c|c|c|c|c|}
\hline \multicolumn{5}{|c|}{ Lesões grandes e extensas do manguito rotador } \\
\hline Variável & & Fileira simples & Fileira dupla & valor-p \\
\hline \multirow[t]{2}{*}{ Elevação } & $\operatorname{ADM}\left({ }^{\circ}\right)$ & $160 \pm 27$ & $164 \pm 26$ & 0,473 \\
\hline & Força $(\mathrm{kg})$ & $3,98 \pm 2,24$ & $6,39 \pm 2,73$ & $0,003^{*}$ \\
\hline \multirow[t]{2}{*}{ Rotação Lateral } & $\operatorname{ADM}\left({ }^{\circ}\right)$ & $62 \pm 22$ & $63 \pm 25$ & 0,845 \\
\hline & Força $(\mathrm{kg})$ & $4,82 \pm 2,33$ & $5,54 \pm 1,66$ & 0,290 \\
\hline \multirow[t]{2}{*}{ Rotação Medial } & $\mathrm{ADM}\left({ }^{\circ}\right)$ & $60 \pm 25$ & $67 \pm 16$ & 0,497 \\
\hline & Força $(\mathrm{kg})$ & $6,23 \pm 2,96$ & $6,50 \pm 2,86$ & 0,546 \\
\hline UCLA & & $33 \pm 3$ & $34 \pm 2$ & 0,148 \\
\hline Constant & & $81 \pm 10$ & $88 \pm 7$ & $0,019^{*}$ \\
\hline
\end{tabular}

Abreviações: ADM, amplitude de movimento; CONSTANT, Constant-Murley Score; UCLA, University of California at Los Angeles Shoulder Rating Scale.

literatura. ${ }^{6,22}$ Esta diferença de idade é importante porque o perfil das rupturas do MR varia bastante de acordo com a faixa etária ${ }^{25}$, sendo que as lesões em idosos são geralmente degenerativas. ${ }^{25}$ Este tipo de lesão, em geral, apresenta maior grau de infiltração gordurosa, variável que se correlaciona com piores desfechos, uma vez que indica má qualidade do tendão e aumenta o risco de rerrupturas. ${ }^{26}$

Quanto ao gênero, ambos os grupos foram compostos por maioria feminina. Este dado vai ao encontro do apresentado pela literatura, que indica que, de forma geral, os pacientes submetidos ao reparo artroscópico do MR são em sua maioria mulheres. ${ }^{2,11,23} \mathrm{Em}$ ambos os grupos, o ombro dominante foi o mais operado, maioria também encontrada em outros estudos nacionais. ${ }^{2,23}$

Estudos biomecânicos enfatizam o potencial aumento da área de contato no footprint e maximização das forças de reparo em $\mathrm{FD}$, o que pode diminuir a taxa de falha anatômica. ${ }^{27,28}$ No entanto, em análise de desfechos clínicos, as controvérsias ainda persistem. ${ }^{7-10}$ Neste sentido, comparamos os resultados de força muscular, função e dor de pacientes após o reparo artroscópico do MR com FS e FD.
Nossos resultados confirmam, em parte, a hipótese inicial. Evidenciamos maior força muscular de elevação anterior em pacientes submetidos a reparo em FD. Porém, as demais variáveis de força muscular, função e dor foram similares entre as técnicas.

Sobhy et al. ${ }^{10}$ concluíram que, a curto (mínimo de 12 meses de seguimento) e médio prazo (mínimo de 24 meses), pacientes tratados com a técnica de FD apresentaram escore UCLA significativamente maior. Além disso, os autores identificaram que, no longo prazo, há uma correlação direta entre a integridade do MR e os resultados funcionais, com superioridade da técnica de FD. Com isso, ao se analisar os resultados funcionais, verifica-se indiretamente a integridade do tendão submetido ao procedimento, reduzindo, em parte, o impacto da não realização de exames de imagem no presente estudo. De forma semelhante, Ying et al. ${ }^{9}$ identificaram valores superiores de força muscular nos pacientes submetidos à técnica de FD. No presente estudo, identificamos maior força muscular no movimento de elevação do ombro nos pacientes que foram submetidos à técnica de FD. No entanto, os resultados de função e dor foram semelhantes 
entre os grupos. A maior área de inserção do tendão no úmero, proporcionado pela técnica de FD, leva a uma maior chance de o tendão reparado manter-se íntegro ao seguimento. Esta integridade está diretamente relacionada ao ganho de força do respectivo músculo. ${ }^{10,22,29,30}$

Ademais, com o objetivo de identificar maiores benefícios para grupos específicos de pacientes, a estratificação por lesões grandes e extensas permitiu a visualização da maior efetividade da técnica de FD nas variáveis de força muscular (elevação anterior do ombro) e do escore Constant. Contudo, não visualizamos diferença estatística nos desfechos analisados ao compararmos as técnicas em lesões pequenas e médias. Quanto às diferenças entre os dois escores utilizados, apesar de o UCLA incluir a força de elevação, ${ }^{16}$ o escore Constant apresenta mais variáveis em sua composição, principalmente uma variedade maior de movimentos e de atividades diárias, ${ }^{17}$ o que pode ter levado aos diferentes resultados entre essas escalas.

Semelhantemente, dois ensaios clínicos randomizados evidenciaram melhores resultados com a técnica de FD em pacientes com lesões $>3 \mathrm{~cm}^{21,31}$ Carbonel et al. ${ }^{21}$ encontraram melhores resultados nos escores UCLA e American Shoulder and Elbow Surgeons (ASES), enquanto Ma et al. ${ }^{31}$ observaram força significativamente maior nos grupos submetidos à FD. Como exposto anteriormente, a técnica de fixação em FD leva a uma maior integridade do tendão no pós-operatório. ${ }^{10,29,30}$ Ao se analisar os fatores que levam a novas rupturas, é bem relatado que o tamanho inicial da lesão é fator significativo de influência sobre a cicatrização do tendão, sendo que o risco relativo de rerruptura aumenta em 2,29 vezes para cada $1 \mathrm{~cm}$ a mais no tamanho da lesão. ${ }^{32,33}$ Esta constatação pode explicar o fato de as lesões maiores se beneficiarem mais da técnica de FD. Uma vez que, naturalmente, tais lesões tendem a apresentar maiores chances de novas lesões, ${ }^{32,33}$ uma técnica que ofereça maior integridade pós-operatória pode trazer melhores resultados ${ }^{10,29,30}$ Porém, em lesões menores, tal benefício não é tão evidente, uma vez que a fixação simples seria suficiente para garantir bons resultados pós-operatórios.

Diante do exposto, conclui-se que a técnica de FD apresenta superioridade apenas na força de elevação do ombro. No entanto, ao se realizar uma análise estratificada pelo tamanho das lesões, a técnica de fixação em FD apresentou melhores resultados para pacientes com lesões $>3 \mathrm{~cm}$ (grandes e extensas). Portanto, parece ser fundamental analisar o tamanho da lesão do paciente ao optar-se por uma técnica de fixação em detrimento da outra.

Quanto aos pontos negativos da FD, além do aumento do custo e do tempo de cirurgia, esta técnica possui complicações específicas, como o local na qual a falha do reparo ocorre. ${ }^{34}$ Enquanto na técnica de FS a falha ocorre no sítio de reparo, na FD ela ocorre na junção musculotendínea, sendo, portanto, uma complicação mais grave, cujo tratamento é desafiador. $^{34}$

Dentre os pontos fortes do presente estudo, destaca-se a análise pós-operatória realizada com diversas variáveis de funcionalidade do ombro, assim como a estratificação dos casos conforme o tamanho da lesão, o que possibilitou identificar um subgrupo de pacientes que apresentaram maior benefício com o uso da técnica de FD.

Dentre as limitações, existe a heterogeneidade quanto à variável idade, o que pode influenciar na análise dos resultados, uma vez que os pacientes submetidos à FS apresentaram média de idade maior. Além disso, cabe destacar a ausência de análise quanto ao custo das técnicas, uma vez que a fixação em FD apresenta maior uso de âncoras. Este é um parâmetro que precisa ser correlacionado com a melhora funcional para se estabelecer o real custo-benefício deste procedimento em estudos futuros.

Ademais, existem limitações quanto ao delineamento do estudo, retrospectivo e observacional, o qual, portanto, não permite concluir quanto à superioridade ou não de uma técnica ou de outra, mas sim levantar hipóteses que devem ser confirmadas por meio de ensaios clínicos. Outra limitação refere-se à não avaliação de fatores prognósticos no préoperatório, como o trofismo muscular e o grau de infiltração gordurosa, assim como a ausência de avaliação de exames de imagem no pós-operatório.

\section{Conclusão}

A utilização da técnica de FD no reparo artroscópico do MR possibilitou melhores resultados funcionais, especialmente nos casos de lesões grandes e extensas, com superioridade na força de elevação anterior e no escore Constant em relação aos pacientes submetidos à técnica de FS.

\section{Suporte Financeiro}

Não houve suporte financeiro de fontes públicas, comerciais, ou sem fins lucrativos.

Conflito de Interesses

Os autores declaram não haver conflito de interesses.

\section{Referências}

1 Sambandam SN, Khanna V, Gul A, Mounasamy V. Rotator cuff tears: An evidence based approach. World J Orthop 2015;6(11): 902-918

2 Carvalho AL, Martinelli F, Tramujas L, Baggio M, Crocetta MS, Martins RO. Rotator cuff injuries and factors associated with reoperation. Rev Bras Ortop 2016;51(03):298-302

3 Dang A, Davies M. Rotator Cuff Disease: Treatment Options and Considerations. Sports Med Arthrosc Rev 2018;26(03):129-133

4 Oliva F, Piccirilli E, Bossa M, et al. I.S.Mu.L.T - Rotator Cuff Tears Guidelines. Muscles Ligaments Tendons J 2016;5(04):227-263

5 Malavolta EA, Assunção JH, Beraldo RA, Pinto GMR, Gracitelli MEC, Ferreira NetoAA. Rotator cuff repair in the Brazilian Unified Health System: Brazilian trends from 2003 to 2015. Rev Bras Ortop 2017;52(04):501-505

6 Vieira FA, Olawa PJ, Belangero PS, Arliani GG, Figueiredo EA, Ejnisman B. Rotator cuff injuries: current perspectives and trends for treatment and rehabilitation. Rev Bras Ortop 2015;50(06): 647-651

7 DeHaan AM, Axelrad TW, Kaye E, Silvestri L, Puskas B, Foster TE. Does double-row rotator cuff repair improve functional outcome of patients compared with single-row technique? A systematic review. Am J Sports Med 2012;40(05):1176-1185

8 Chen M, Xu W, Dong Q, Huang Q, Xie Z, Mao Y. Outcomes of singlerow versus double-row arthroscopic rotator cuff repair: a 
systematic review and meta-analysis of current evidence. Arthroscopy 2013;29(08):1437-1449

9 Ying ZM, Lin T, Yan SG. Arthroscopic single-row versus doublerow technique for repairing rotator cuff tears: a systematic review and meta-analysis. Orthop Surg 2014;6(04):300-312

10 Sobhy MH, Khater AH, Hassan MR, El Shazly O. Do functional outcomes and cuff integrity correlate after single- versus doublerow rotator cuff repair? A systematic review and meta-analysis study. Eur J Orthop Surg Traumatol 2018;28(04):593-605

11 Senna LF, Ramos MRF, Bergamaschi RF. Arthroscopic rotator cuff repair: single-row $v s$. double-row - clinical results after one to four years. Rev Bras Ortop 2018;53(04):448-453

12 Huang AL, Thavorn K, van Katwyk S, MacDonald P, Lapner P. Double-Row Arthroscopic Rotator Cuff Repair Is More Cost-Effective Than Single-Row Repair. J Bone Joint Surg Am 2017;99(20): $1730-1736$

13 Lo IK, Burkhart SS. Double-row arthroscopic rotator cuff repair: re-establishing the footprint of the rotator cuff. Arthroscopy 2003;19(09):1035-1042

14 Davidson J, Burkhart SS. The geometric classification of rotator cuff tears: a system linking tear pattern to treatment and prognosis. Arthroscopy 2010;26(03):417-424

15 Andrade RP, Correa Filho MRC, Queiroz BC. Lesões do manguito rotador. Rev Bras Ortop 2004;39(11/12):621-635

16 Amstutz HC, Sew Hoy AL, Clarke IC. UCLA anatomic total shoulder arthroplasty. Clin Orthop Relat Res 1981;(155):7-20

17 Constant CR, Murley AH. A clinical method of functional assessment of the shoulder. Clin Orthop Relat Res 1987;(214):160-164

18 Scott J, Huskisson EC. Graphic representation of pain. Pain 1976;2 (02):175-184

19 Barreto RP, Barbosa ML, Balbinotti MA, Mothes FC, da Rosa LH, Silva MF. The Brazilian version of the Constant-Murley Score (CMS-BR): convergent and construct validity, internal consistency, and unidimensionality. Rev Bras Ortop 2016;51(05):515-520

20 Oku EC, Andrade AP, Stadiniky SP, Carrera EF, Tellini GG. Tradução e adaptação cultural do Modified-University of California at Los Angeles Shoulder Rating Scale para a língua portuguesa. Rev Bras Reumatol 2006;46(04):246-252

21 Carbonel I, Martinez AA, Calvo A, Ripalda J, Herrera A. Single-row versus double-row arthroscopic repair in the treatment of rotator cuff tears: a prospective randomized clinical study. Int Orthop 2012;36(09):1877-1883
22 Godinho GG, França FdeO, Freitas JM, et al. Result from arthroscopic surgical treatment of renewed tearing of the rotator cuff of the shoulder. Rev Bras Ortop 2015;50(01):89-93

23 Miyazaki AN, da Silva LA, Santos PD, Checchia SL, Cohen C, Giora TS. Evaluation of the results from arthroscopic surgical treatment of rotator cuff injuries in patients aged 65 years and over. Rev Bras Ortop 2015;50(03):305-311

24 Gilotra M, O’Brien MJ, Savoie FH 3rd. Arthroscopic Rotator Cuff Repair: Indication and Technique. Instr Course Lect 2016; 65:83-92

25 Lazarides AL, Alentorn-Geli E, Choi JH, et al. Rotator cuff tears in young patients: a different disease than rotator cuff tears in elderly patients. J Shoulder Elbow Surg 2015;24(11):1834-1843

26 McElvany MD, McGoldrick E, Gee AO, Neradilek MB, Matsen FA 3rd. Rotator cuff repair: published evidence on factors associated with repair integrity and clinical outcome. Am J Sports Med 2015; 43(02):491-500

27 Meier SW, Meier JD. The effect of double-row fixation on initial repair strength in rotator cuff repair: a biomechanical study. Arthroscopy 2006;22(11):1168-1173

28 Smith CD, Alexander S, Hill AM, et al. A biomechanical comparison of single and double-row fixation in arthroscopic rotator cuff repair. J Bone Joint Surg Am 2006;88(11):2425-2431

29 Tudisco C, Bisicchia S, Savarese E, et al. Single-row vs. double-row arthroscopic rotator cuff repair: clinical and 3 Tesla MR arthrography results. BMC Musculoskelet Disord 2013;14:43

30 Shen C, Tang ZH, Hu JZ, Zou GY, Xiao RC. Incidence of retear with double-row versus single-row rotator cuff repair. Orthopedics 2014;37(11):e1006-e1013

31 Ma HL, Chiang ER, Wu HT, et al. Clinical outcome and imaging of arthroscopic single-row and double-row rotator cuff repair: a prospective randomized trial. Arthroscopy 2012;28(01): $16-24$

32 Nho SJ, Brown BS, Lyman S, Adler RS, Altchek DW, MacGillivray JD. Prospective analysis of arthroscopic rotator cuff repair: prognostic factors affecting clinical and ultrasound outcome. J Shoulder Elbow Surg 2009;18(01):13-20

33 Bedeir YH, Jimenez AE, Grawe BM. Recurrent tears of the rotator cuff: Effect of repair technique and management options. Orthop Rev (Pavia) 2018;10(02):7593

34 Schmidt CC, Jarrett CD, Brown BT. Management of rotator cuff tears. J Hand Surg Am 2015;40(02):399-408 